\title{
On a sharp lemma of Cassels and Montgomery on manifolds
}

\author{
Luca Brandolini $^{1}\left[\right.$ - Bianca Gariboldi ${ }^{1} \cdot$ Giacomo Gigante $^{1}$ \\ Received: 20 March 2020 / Revised: 26 October 2020 / Accepted: 3 November 2020 / \\ Published online: 21 November 2020 \\ (c) The Author(s) 2020
}

\section{Abstract}

Let $(\mathcal{M}, g)$ be a $d$-dimensional compact connected Riemannian manifold and let $\left\{\varphi_{m}\right\}_{m=0}^{+\infty}$ be a complete sequence of orthonormal eigenfunctions of the LaplaceBeltrami operator on $\mathcal{M}$. We show that there exists a positive constant $C$ such that for all integers $N$ and $X$ and for all finite sequences of $N$ points in $\mathcal{M},\{x(j)\}_{j=1}^{N}$, and positive weights $\left\{a_{j}\right\}_{j=1}^{N}$ we have

$$
\sum_{m=0}^{X}\left|\sum_{j=1}^{N} a_{j} \varphi_{m}(x(j))\right|^{2} \geq \max \left\{C X \sum_{j=1}^{N} a_{j}^{2},\left(\sum_{j=1}^{N} a_{j}\right)^{2}\right\}
$$

Mathematics Subject Classification 41A55 - 11K38

Let $(\mathcal{M}, g)$ be a $d$-dimensional compact connected Riemannian manifold, with normalized Riemannian measure $\mu$ such that $\mu(\mathcal{M})=1$, and Riemannian distance $d(x, y)$. Let $\left\{\lambda_{m}^{2}\right\}_{m=0}^{+\infty}$ be the sequence of eigenvalues of the (positive) LaplaceBeltrami operator $\Delta$, listed in increasing order with repetitions, and let $\left\{\varphi_{m}\right\}_{m=0}^{+\infty}$ be an associated sequence of orthonormal eigenfunctions. In particular $\varphi_{0} \equiv 1$ and $\lambda_{0}=0$.

Communicated by Loukas Grafakos.

The authors have been supported by a GNAMPA 2019 project.

Luca Brandolini

luca.brandolini@unibg.it

Bianca Gariboldi

biancamaria.gariboldi@unibg.it

Giacomo Gigante

giacomo.gigante@unibg.it

1 Dipartimento di Ingegneria Gestionale, dell'Informazione e della Produzione, Università degli Studi di Bergamo, Viale Marconi 5, Dalmine BG, Italy 
This allows to define the Fourier coefficients of $L^{1}(\mathcal{M})$ functions as

$$
\widehat{f}\left(\lambda_{m}\right)=\int_{\mathcal{M}} f(x) \overline{\varphi_{m}(x)} d \mu(x)
$$

and the associated Fourier series

$$
\sum_{m=0}^{+\infty} \widehat{f}\left(\lambda_{m}\right) \varphi_{m}(x)
$$

The main result of this paper is the following theorem.

Theorem 1 There exists a positive constant $C$ such that for all integers $N$ and $X$ and for all finite sequences of $N$ points in $\mathcal{M},\{x(j)\}_{j=1}^{N}$, and positive weights $\left\{a_{j}\right\}_{j=1}^{N}$ we have

$$
\sum_{m=0}^{X}\left|\sum_{j=1}^{N} a_{j} \varphi_{m}(x(j))\right|^{2} \geq \max \left\{C X \sum_{j=1}^{N} a_{j}^{2},\left(\sum_{j=1}^{N} a_{j}\right)^{2}\right\} .
$$

Notice that the estimate

$$
\sum_{m=0}^{X}\left|\sum_{j=1}^{N} a_{j} \varphi_{m}(x(j))\right|^{2} \geq\left(\sum_{j=1}^{N} a_{j}\right)^{2}
$$

is immediately obtained since for $m=0$ one has $\varphi_{0}(x)=1$ for all $x$ in $\mathcal{M}$. The essential part of the theorem is therefore the estimate

$$
\sum_{m=0}^{X}\left|\sum_{j=1}^{N} a_{j} \varphi_{m}(x(j))\right|^{2} \geq C X \sum_{j=1}^{N} a_{j}^{2} .
$$

Since for any $m$ the expected value of $\left|\sum a_{j} \varphi_{m}(x(j))\right|^{2}$ is $\sum a_{j}^{2}$ (see the proof of Proposition 2 below) the above estimate means that independently of how the points are chosen, there is a positive proportion of values of $m$ between 0 and $X$ for which $\left|\sum a_{j} \varphi_{m}(x(j))\right|^{2}$ cannot be essentially smaller than its expected value.

When $\mathcal{M}$ is the one-dimensional torus, the above theorem is classical and goes back to the work of Cassels [5]. He was interested in estimates on exponential sums, and their relation to Dirichlet's approximation theorem. More precisely, as part of the proof of a slightly weaker version of Dirichlet's theorem, in [5, page 288] he showed that for any choice of $N$ real numbers $x(1), \ldots, x(N)$ and for any integer $p \geq 1$,

$$
\sum_{m=1}^{X}\left|\sum_{j=1}^{N} e^{2 \pi i m x(j)}\right|^{2 p} \geq \frac{1}{2}(X+1) C-\frac{1}{2} N^{2 p},
$$


where $C$ is the sum of the squares of the coefficients in the usual multinomial expansion of $(x(1)+\cdots+x(N))^{p}$. When $p=1$, we have $C=N$ and Cassels estimate reduces to a version of our estimate (1) for the torus and for $a_{j}=1$. In [14, Theorem 8, Chapter 5], Montgomery gave a version of (3) (with $p=1$ ) with positive weights,

$$
\sum_{m=1}^{X}\left|\sum_{j=1}^{N} a_{j} e^{2 i m x(j)}\right|^{2} \geq \frac{1}{2}(X+1) \sum_{j=1}^{N} a_{j}^{2}-\frac{1}{2}\left(\sum_{j=1}^{N} a_{j}\right)^{2} .
$$

He also proved the following version of Cassels inequality on the two dimensional torus $\mathbb{T}^{2}\left(\left[14\right.\right.$, Theorem 12 in Chapter 5]). For any $\mathbf{x}(1), \ldots \mathbf{x}(N)$ in $\mathbb{T}^{2}$ and for any $X_{1}, X_{2}$

$$
\sum_{\left|m_{1}\right| \leq X_{1},\left|m_{2}\right| \leq X_{2}, \mathbf{m} \neq \mathbf{0}}\left|\sum_{j=1}^{N} e^{2 \pi i \mathbf{m} \cdot \mathbf{x}(j)}\right|^{2} \geq N X_{1} X_{2}-N^{2} .
$$

The proof of these results was inspired by Siegel's analytic proof of Minkowski's convex body theorem [15]. Indeed, given a symmetric convex body $\mathcal{C}$, there exists a non-negative trigonometric polynomial $T$ such that $\widehat{T}$ is also non-negative, the support of $\widehat{T}$ is contained in $\mathcal{C}, T(\mathbf{0}) \geq \operatorname{area}(\mathcal{C}) / 4$ and $\widehat{T}(\mathbf{0})=1$. It follows that

$$
\begin{aligned}
& \sum_{\mathbf{m} \in \mathcal{C}}\left|\sum_{j=1}^{N} e^{2 \pi i \mathbf{m} \cdot \mathbf{x}(j)}\right|^{2} \geq \sum_{\mathbf{m} \in \mathcal{C}} \widehat{T}(\mathbf{m})\left|\sum_{j=1}^{N} e^{2 \pi i \mathbf{m} \cdot \mathbf{x}(j)}\right|^{2} \\
& \quad=\sum_{j=1}^{N} \sum_{k=1}^{N} \sum_{\mathbf{m} \in \mathcal{C}} \widehat{T}(\mathbf{m}) e^{2 \pi i \mathbf{m} \cdot(\mathbf{x}(j)-\mathbf{x}(k))}=\sum_{j=1}^{N} \sum_{k=1}^{N} T(\mathbf{x}(j)-\mathbf{x}(k)) \\
& \geq N T(\mathbf{0}) \geq N \operatorname{area}(\mathcal{C}) / 4 .
\end{aligned}
$$

The construction of the trigonometric polynomial $T$ is the following. Let $\overline{\mathbf{x}}$ such that $\sharp\left(\left(\frac{1}{2} \mathcal{C}-\overline{\mathbf{x}}\right) \cap \mathbb{Z}^{2}\right) \geq$ area $(\mathcal{C}) / 4$. Then one can simply take

$$
T(\mathbf{x})=\frac{1}{\sharp\left(\left(\frac{1}{2} \mathcal{C}-\overline{\mathbf{x}}\right) \cap \mathbb{Z}^{2}\right)} \sum_{\mathbf{m}, \mathbf{k} \in \frac{1}{2} \mathcal{C}-\overline{\mathbf{x}}} e^{2 \pi i(\mathbf{m}-\mathbf{k}) \cdot \mathbf{x}} .
$$

The applications that Montgomery had in mind for this type of inequalities was to the theory of irregularities of distribution (see [14, Chapter 6]. See also [20]). Let $\mathcal{C}$ be a subset of $\mathbb{T}^{2}$ and for any collection of $N$ points $\mathbf{x}(1), \ldots, \mathbf{x}(N)$ define the discrepancy function

$$
D_{\mathcal{C}}(\mathbf{x})=\sum_{j=1}^{N} \chi_{\mathcal{C}}(\mathbf{x}+\mathbf{x}(j))-\operatorname{area}(\mathcal{C})
$$


By Parseval's identity, its $L^{2}$ norm is

$$
\int_{\mathbb{T}^{2}}\left|D_{\mathcal{C}}(\mathbf{x})\right|^{2} d \mathbf{x}=\sum_{\mathbf{m} \neq \mathbf{0}}\left|\widehat{\chi}_{\mathcal{C}}(\mathbf{m})\right|^{2}\left|\sum_{j=1}^{N} e^{2 \pi i \mathbf{m} \cdot \mathbf{x}(j)}\right|^{2}
$$

The idea is now to estimate this quantity from below by means of inequality (4). In general, due to the zeros of the function $\widehat{\chi}_{\mathcal{C}}$, a lower bound for $\left|\widehat{\chi}_{\mathcal{C}}(\mathbf{m})\right|^{2}$ is not available. However, it is sometimes possible to let $\mathcal{C}$ vary in some class of subsets of $\mathbb{T}^{2}$, in such a way that the average of the Fourier coefficients $\left|\widehat{\chi}_{\mathcal{C}}(\mathbf{m})\right|$ over this collection is bounded below by some decreasing function of $|\mathbf{m}|$. For example, one can take two disks of radius $1 / 4$ and $1 / 2$, or one can take dilations of a square, or rotations and dilations of a smooth convex set. In the case of two disks $\mathcal{C}_{1}$ and $\mathcal{C}_{2}$ of radius $1 / 4$ and $1 / 2$ respectively, Montgomery showed that

$$
\left|\widehat{\chi}_{\mathcal{C}_{1}}(\mathbf{m})\right|^{2}+\left|\widehat{\chi}_{\mathcal{C}_{2}}(\mathbf{m})\right|^{2} \geq c \frac{1}{|\mathbf{m}|^{3}}
$$

Thus,

$$
\int_{\mathbb{T}^{2}}\left|D_{\mathcal{C}_{1}}(\mathbf{x})\right|^{2} d \mathbf{x}+\int_{\mathbb{T}^{2}}\left|D_{\mathcal{C}_{2}}(\mathbf{x})\right|^{2} d \mathbf{x} \geq c \sum_{\mathbf{m} \neq \mathbf{0}} \frac{1}{|\mathbf{m}|^{3}}\left|\sum_{j=1}^{N} e^{2 \pi i \mathbf{m} \cdot \mathbf{x}(j)}\right|^{2}
$$

Applying now inequality (4) with $X_{1}=X_{2}=(2 N)^{1 / 2}$, this is bounded below by $c N X_{1} X_{2}\left(\sqrt{2} X_{1}\right)^{-3}=c N^{1 / 2}$. This means that for any choice of $N$ points there is a disk of radius $1 / 4$ or $1 / 2$ for which the discrepancy is greater than $c N^{1 / 4}$.

This type of arguments have a straightforward extension to the higher dimensional torus.

If the collection of points $\{\mathbf{x}(j)\}_{j=1}^{N}$ in $\mathbb{T}^{d}$ is not evenly distributed, one has to expect that the lower bound in (5) is not achieved (e.g., let all points be the same). This suggests that the exponential sums

$$
\sum_{\mathbf{m} \in \mathcal{C}}\left|\sum_{j=1}^{N} e^{2 \pi i \mathbf{m} \cdot \mathbf{x}(j)}\right|^{2}
$$

can be used as a measure of the regularity of the collection of points. See [13] where this is related with Riesz type energy functionals for the torus.

A spherical analog of Cassels-Montgomery inequality has also been used by Bilyk and Dai [2, formula (4.9)] to prove a lower bound for the discrepancy in the $d$ dimensional sphere.

Recently, Bilyk, Dai, Steinerberger [3] extended Cassels-Montgomery inequality to the case of smooth compact $d$-dimensional Riemannian manifolds without boundary. More precisely they showed that there exists a positive constant $C$ such that for all 
integers $N$ and $X$ and for all finite sequences of $N$ points in $\mathcal{M},\{x(j)\}_{j=1}^{N}$, and positive weights $\left\{a_{j}\right\}_{j=1}^{N}$,

$$
\sum_{m=0}^{X}\left|\sum_{j=1}^{N} a_{j} \varphi_{m}(x(j))\right|^{2} \geq C \frac{X}{(\log X)^{d / 2}} \sum_{j=1}^{N} a_{j}^{2}
$$

This result should be compared with the following simple proposition.

Proposition 2 Let $X$ and $N$ be positive integers. For all positive weights $\left\{a_{j}\right\}_{j=1}^{N}$, there exists a sequence of points $\{x(j)\}_{j=1}^{N}$ in $\mathcal{M}$ such that

$$
\sum_{m=0}^{X}\left|\sum_{j=1}^{N} a_{j} \varphi_{m}(x(j))\right|^{2} \leq X \sum_{j=1}^{N} a_{j}^{2}+\left(\sum_{j=1}^{N} a_{j}\right)^{2}
$$

\section{Proof Let}

$$
\Phi\left(y_{1}, \ldots, y_{N}\right)=\sum_{m=1}^{X}\left|\sum_{j=1}^{N} a_{j} \varphi_{m}\left(y_{j}\right)\right|^{2}=\sum_{m=1}^{X} \sum_{j, k=1}^{N} a_{j} a_{k} \varphi_{m}\left(y_{j}\right) \overline{\varphi_{m}\left(y_{k}\right)}
$$

Since for $m \neq 0$

$$
\int_{\mathcal{M}} \varphi_{m}\left(y_{j}\right) d y_{j}=0
$$

if $j \neq k$ we have

$$
\int_{\mathcal{M}} \cdots \int_{\mathcal{M}} \varphi_{m}\left(y_{j}\right) \overline{\varphi_{m}\left(y_{k}\right)} d y_{1} \cdots d y_{N}=\int_{\mathcal{M}} \int_{\mathcal{M}} \varphi_{m}\left(y_{j}\right) \overline{\varphi_{m}\left(y_{k}\right)} d y_{j} d y_{k}=0
$$

while

$$
\int_{\mathcal{M}} \cdots \int_{\mathcal{M}} \varphi_{m}\left(y_{j}\right) \overline{\varphi_{m}\left(y_{j}\right)} d y_{1} \cdots d y_{N}=\int_{\mathcal{M}}\left|\varphi_{m}\left(y_{j}\right)\right|^{2} d y_{j}=1
$$

Hence,

$$
\begin{aligned}
& \int_{\mathcal{M}} \cdots \int_{\mathcal{M}} \Phi\left(y_{1}, \ldots, y_{N}\right) d y_{1} \cdots d y_{N} \\
& =\sum_{m=1}^{X} \sum_{j=1}^{N} a_{j}^{2} \int_{\mathcal{M}}\left|\varphi_{m}\left(y_{j}\right)\right|^{2} d y_{j}=X \sum_{j=1}^{N} a_{j}^{2} .
\end{aligned}
$$


Therefore there exist points $\{x(j)\}_{j=1}^{N}$ such that

$$
\Phi(x(1), \ldots, x(N)) \leq X \sum_{j=1}^{N} a_{j}^{2} .
$$

Our goal is therefore to remove the logarithmic loss in the above result of Bilyk, Dai and Steinerberger, thus obtaining a sharp estimate.

The original proof by Montgomery in the case of the torus uses the Fejér kernel, or more in general a trigonometric polynomial as the one described in (6). A direct adaptation of this proof to the case of a general manifold would require to construct a positive kernel of the form

$$
\sum_{m=0}^{X} c_{m} \varphi_{m}(x) \overline{\varphi_{m}(y)}
$$

but unfortunately this type of kernel is not available in a general manifold. One could therefore withdraw, for example, the requirement that the spectrum of the kernel be contained in the set $\left\{\lambda_{0}^{2}, \ldots, \lambda_{X}^{2}\right\}$. This is the strategy followed by Bilyk, Dai and Steinerberger which use the heat kernel. Our strategy here is on the contrary to use a kernel which is positive up to a negligible error, without dropping the spectrum condition. The existence of such type of kernel can be proved by means of the Hadamard parametrix for the wave operator on the manifold. In the next section we introduce this construction.

A simple consequence of Theorem 1 is the following estimate on the maximum degree $X$ of linear combinations of eigenfunctions of the Laplacian up to the eigenvalue $\lambda_{X}$ that a quadrature rule can integrate exactly. This is a well known result for equal weights, see e.g. [8, Proposition 1], or [18, Theorem 2] where one can find an estimate of the constant $C$ that depends only on the dimension of the manifold. See also [18, Theorem 1] for a result with general weights.

Corollary 3 Let $X$ be a positive integer and assume there exist points $\{x(j)\}_{j=1}^{N}$ and weights $\left\{a_{j}\right\}_{j=1}^{N}$ such that for every polynomial

$$
P(x)=\sum_{m=0}^{X} c_{m} \varphi_{m}(x)
$$

we have

$$
\int_{\mathcal{M}} P(x) d x=\sum_{j=1}^{N} a_{j} P(x(j)) .
$$


Then there exists a constant $C>0$ independent of $X$ and $N$ such that

$$
1 \geq C X \sum_{j=1}^{N} a_{j}^{2}
$$

In particular

$$
C X \leqslant N \text {. }
$$

Proof Since $\varphi_{0}(x) \equiv 1$ we must have $\sum_{i=1}^{N} a_{i}=1$. Let

$$
P(x)=\sum_{m=0}^{X} \sum_{i=1}^{N} a_{i} \overline{\varphi_{m}(x(i))} \varphi_{m}(x),
$$

then

$$
\begin{aligned}
\int_{\mathcal{M}} P(x) d x & =\int_{\mathcal{M}} \sum_{m=0}^{X} \sum_{i=1}^{N} a_{i} \overline{\varphi_{m}(x(i))} \varphi_{m}(x) d x \\
& =\sum_{m=0}^{X} \sum_{i=1}^{N} a_{i} \overline{\varphi_{m}(x(i))} \int_{\mathcal{M}} \varphi_{m}(x) d x=\sum_{i=1}^{N} a_{i}=1 .
\end{aligned}
$$

On the other hand

$$
\begin{aligned}
\sum_{j=1}^{N} a_{j} P(x(j)) & =\sum_{j=1}^{N} a_{j} \sum_{m=0}^{X} \sum_{i=1}^{N} a_{i} \overline{\varphi_{m}(x(i))} \varphi_{m}(x(j)) \\
& =\sum_{m=0}^{X}\left|\sum_{j=1}^{N} a_{j} \varphi_{m}(x(j))\right|^{2} \geq C X \sum_{j=1}^{N} a_{j}^{2} .
\end{aligned}
$$

Hence

$$
1 \geq C X \sum_{j=1}^{N} a_{j}^{2}
$$

Applying Cauchy-Schwarz inequality to $1=\sum_{i=1}^{N} a_{i}$ we easily obtain

$$
\sum_{j=1}^{N} a_{j}^{2} \geqslant 1 / N
$$

and therefore $N \geq C X$. 
When the manifold $\mathcal{M}$ is the $d$ dimensional sphere and the weights $a_{j}$ are all equal, the existence of point distributions (spherical designs) $\{x(j)\}_{j=1}^{N(X)}$ for which (7) holds for some $N(X) \leq c X$ is the famous conjecture of Korevaar and Meyers [12], recently proved by Bondarenko, Radchenko, Viazovska [4]. The analogous result for a general manifold has been proved in [8]. See also [6] for the case of general weights.

\section{The Hadamard parametrix for the wave equation}

Following [11, III, §17.4], for $v=0,1,2, \ldots$, let us call $E_{v}(t, x)$ the distribution defined as the inverse Fourier-Laplace transform on $\mathbb{R}^{d+1}$ of $\nu !\left(|\xi|^{2}-\tau^{2}\right)^{-\nu-1}$,

$$
E_{\nu}(t, x)=\nu !(2 \pi)^{-d-1} \int_{\operatorname{Im} \tau=c<0} e^{i(x \cdot \xi+t \tau)}\left(|\xi|^{2}-\tau^{2}\right)^{-\nu-1} d \xi d \tau
$$

Note that for $v=0$, this is exactly the fundamental solution of the wave operator, see $[11$, I, §6.2]. The next Proposition (see [11, III, Lemma 17.4.2]) gives more information about the distributions $E_{v}$.

Proposition 4 (i) $E_{v}$ is a homogeneous distribution of degree $2 v-d+1$ supported in the forward light cone $\left\{(t, x) \in \mathbb{R}^{1+d}: t>0, t^{2} \geq|x|^{2}\right\}$.

(ii) Moreover

$$
E_{\nu}(t, x)=2^{-2 v-1} \pi^{(1-d) / 2} \chi_{+}^{\nu+(1-d) / 2}\left(t^{2}-|x|^{2}\right), \quad t>0
$$

and $E_{v}(t, x)$ can be regarded as a smooth function of $t>0$ with values in $\mathcal{D}^{\prime}\left(\mathbb{R}^{d}\right)$. In particular if $\psi \in C_{0}^{\infty}\left(\mathbb{R}^{1+d}\right)$ then

$$
\left\langle E_{\nu}, \psi\right\rangle=2^{-2 \nu-1} \pi^{(1-d) / 2} \int_{0}^{+\infty}\left\langle\chi_{+}^{\nu+(1-d) / 2}\left(t^{2}-|\cdot|^{2}\right), \psi(t, \cdot)\right\rangle d t
$$

Also

$$
\partial_{t}^{k} E_{v}\left(0^{+}, \cdot\right)=0 \text { for } k \leq 2 v
$$

and

$$
\partial_{t}^{2 v+1} E_{v}\left(0^{+}, \cdot\right)=v ! \delta_{0} .
$$

(iii) Finally, setting

$$
\left\langle\check{E}_{\nu}, \varphi\right\rangle:=\left\langle E_{\nu}, \check{\varphi}\right\rangle,
$$

where

$$
\check{\varphi}(t, x)=\varphi(-t, x),
$$


the distributions $\left(E_{v}-\check{E}_{v}\right)(t, x)$ and $\partial_{t}\left(E_{v}-\check{E}_{v}\right)(t, x)$ can be regarded as continuous radial functions of $x$ with values in $\mathcal{D}^{\prime}(\mathbb{R})$. With a small abuse of notation we will write $\left(E_{v}-\check{E}_{v}\right)(\cdot,|x|)$ and $\partial .\left(E_{v}-\check{E}_{v}\right)(\cdot,|x|)$.

Let us clarify the meaning of the objects that appear in this proposition. Let $\alpha \in \mathbb{C}$ be such that $\operatorname{Re} \alpha>-1$ and for every test function $\varphi \in C_{0}^{\infty}(\mathbb{R})$ define the distribution $\chi_{+}^{\alpha}$ as

$$
\left\langle\chi_{+}^{\alpha}, \varphi\right\rangle=\frac{1}{\Gamma(\alpha+1)} \int_{0}^{+\infty} x^{\alpha} \varphi(x) d x
$$

Integration by parts immediately gives

$$
\left\langle\chi_{+}^{\alpha}, \varphi\right\rangle=-\left\langle\chi_{+}^{\alpha+1}, \varphi^{\prime}\right\rangle
$$

so that $\chi_{+}^{\alpha}$ can be extended to all $\alpha$ with $\operatorname{Re} \alpha>-2$, and, repeating the argument, to the whole complex plane (see [11, I, §3.2] for the details).

Also, since the function $f(x, t)=t^{2}-|x|^{2}$ is a submersion of $\mathbb{R}^{d+1} \backslash\{0\}$ in $\mathbb{R}$, then the pull-back $\chi_{+}^{\alpha}\left(t^{2}-|x|^{2}\right):=f^{*}\left(\chi_{+}^{\alpha}\right) \in \mathcal{D}^{\prime}\left(\mathbb{R}^{d+1} \backslash\{0\}\right)$ is defined by the identity

$$
\left\langle f^{*}\left(\chi_{+}^{\alpha}\right), \varphi\right\rangle:=\left\langle\chi_{+}^{\alpha}, \int_{f^{-1}(\cdot)} \frac{\varphi(x, t)}{\|\nabla f(x, t)\|} d \sigma(x, t)\right\rangle .
$$

We observe that by [11, I, Theorem 3.23] the distribution $\chi_{+}^{\nu+(1-d) / 2}\left(t^{2}-|x|^{2}\right)$ can be uniquely extended to $\mathcal{D}^{\prime}\left(\mathbb{R}^{d+1}\right)$ for $v=0,1, \ldots$.

Recall that distributions in $\mathcal{D}^{\prime}(\mathcal{M})$ can always be written as $u=\sum_{m=0}^{+\infty} c_{m} \varphi_{m}$, where the sequence $\left\{c_{m}\right\}$ is slowly increasing. Their action on smooth functions is given by

$$
\langle u, \phi\rangle=\sum_{m=0}^{+\infty} c_{m} \int \phi \varphi_{m} .
$$

Consider the continuous linear map $\mathcal{K}_{t}: \mathcal{D}(\mathcal{M}) \rightarrow \mathcal{D}^{\prime}(\mathcal{M})$ defined by

$$
\phi \mapsto \mathcal{K}_{t} \phi=\sum_{m} \cos \left(\lambda_{m} t\right) \widehat{\phi}\left(\lambda_{m}\right) \varphi_{m}
$$

Observe that $\mathcal{K}_{t} \phi$ is in fact a smooth function and it is the solution of the following Cauchy problem for the wave equation

$$
\left\{\begin{array}{l}
\left(\frac{\partial^{2}}{\partial t^{2}}+\Delta_{x}\right) w(t, x)=0 \\
w(0, x)=\phi(x) \\
\frac{\partial w}{\partial t}(0, x)=0
\end{array}\right.
$$


By the Schwartz kernel Theorem (see [11, I, Theorem 5.2.1]), there exists one and only one distribution $\cos (t \sqrt{\Delta})(x, y) \in \mathcal{D}^{\prime}(\mathcal{M} \times \mathcal{M})$, called kernel of the map $\mathcal{K}_{t}$, such that

$$
\begin{aligned}
\langle\cos (t \sqrt{\Delta})(x, y), \eta(x) \phi(y)\rangle & =\left\langle\mathcal{K}_{t} \phi, \eta\right\rangle=\sum_{m} \cos \left(\lambda_{m} t\right) \widehat{\phi}\left(\lambda_{m}\right)\left\langle\varphi_{m}, \eta\right\rangle \\
& =\sum_{m} \cos \left(\lambda_{m} t\right) \int \phi(y) \overline{\varphi_{m}(y)} d y \int \eta(x) \varphi_{m}(x) d x .
\end{aligned}
$$

This immediately implies that

$$
\cos (t \sqrt{\Delta})(x, y)=\sum_{m} \cos \left(\lambda_{m} t\right) \varphi_{m}(x) \overline{\varphi_{m}(y)},
$$

and the identity is of course in the sense of distributions in $\mathcal{D}^{\prime}(\mathcal{M} \times \mathcal{M})$.

Hadamard's construction of the parametrix for the wave operator allows to describe for small values of time $t$ the singularities of $\cos (t \sqrt{\Delta})(x, y)$.

Theorem 5 (see [16, Theorem 3.1.5]) Given a d-dimensional Riemannian manifold $(\mathcal{M}, g)$, there exists $\varepsilon>0$ and functions $\alpha_{v} \in \mathcal{C}^{\infty}(\mathcal{M} \times \mathcal{M})$, so that if $Q>d+3$ the following holds. Let

$$
K_{Q}(t, x, y)=\sum_{\nu=0}^{Q} \alpha_{\nu}(x, y) \partial_{t}\left(E_{v}-\check{E}_{v}\right)(t, d(x, y))
$$

and

$$
R_{Q}(t, x, y)=\cos (t \sqrt{\Delta})(x, y)-K_{Q}(t, x, y),
$$

then $R_{Q} \in \mathcal{C}^{Q-d-3}([-\varepsilon, \varepsilon] \times \mathcal{M} \times \mathcal{M})$ and

$$
\left|\partial_{t, x, y}^{\beta} R_{Q}(t, x, y)\right| \leq C|t|^{2 Q+2-d-|\beta|} .
$$

Furthermore $\alpha_{0}(x, y)>0$ in $\mathcal{M} \times \mathcal{M}$.

Observe that $K_{Q}(t, x, y)$, by Proposition 4 (iii), defines a distribution on $\mathbb{R} \times \mathcal{M} \times$ $\mathcal{M}$ via the identity

$$
\left\langle K_{Q}, \varphi\right\rangle=\iint_{\mathcal{M} \times \mathcal{M}}\left\langle K_{Q}(\cdot, x, y), \varphi(\cdot, x, y)\right\rangle d x d y .
$$

However this distribution describes the singularities of the kernel $\cos (t \sqrt{\Delta})(x, y)$ only for small time. 


\section{Notations and Fourier transforms}

Let us introduce some notation. If $f$ and $g$ are integrable functions on $\mathbb{R}^{d}$, we shall denote their convolution by

$$
f *_{d} g(x)=\int_{\mathbb{R}^{d}} f(x-y) g(y) d y .
$$

We define the cosine transform of smooth even functions on $\mathbb{R}$ as

$$
\mathcal{C} f(t)=\int_{0}^{\infty} f(s) \cos (s t) d s
$$

with inverse

$$
\mathcal{C}^{-1} f(s)=\frac{2}{\pi} \int_{0}^{\infty} f(t) \cos (s t) d t
$$

For smooth functions on $\mathbb{R}^{d}$ we will use a slightly different normalization, and we define the Fourier transform and its inverse as

$$
\begin{aligned}
\mathcal{F}_{d} f(\xi) & =\int_{\mathbb{R}^{d}} f(x) e^{-2 \pi i x \cdot \xi} d x, \\
\mathcal{F}_{d}^{-1} f(x) & =\int_{\mathbb{R}^{d}} f(\xi) e^{2 \pi i x \cdot \xi} d \xi .
\end{aligned}
$$

For radial functions $f(x)=f_{0}(|x|)$, the above Fourier transform reduces essentially to the Hankel transform, given by (see [17, Chapter 4, Theorem 3.3])

$$
\begin{gathered}
\mathcal{F}_{d} f(\xi)=2 \pi|\xi|^{-\frac{d-2}{2}} \int_{0}^{\infty} f_{0}(s) J_{\frac{d-2}{2}}(2 \pi|\xi| s) s^{\frac{d}{2}} d s \\
\mathcal{F}_{d}^{-1} f(x)=2 \pi|x|^{-\frac{d-2}{2}} \int_{0}^{\infty} f_{0}(s) J_{\frac{d-2}{2}}(2 \pi|x| s) s^{\frac{d}{2}} d s .
\end{gathered}
$$

In the future, with an abuse of notation, we will identify the function $f$ with its radial profile $f_{0}$ and write $\mathcal{F}_{d} f(|\xi|)$ instead of $\mathcal{F}_{d} f(\xi)$. One can easily show that

$$
\mathcal{F}_{1} f(t)=2 \mathcal{C} f(2 \pi t)
$$

In the proof of Theorem 1 we need the inverse cosine transform of the distribution $\partial_{t}\left(E_{v}-\breve{E}_{v}\right)$. By Proposition 4 (iii), $\partial_{t}\left(E_{v}-\check{E}_{v}\right)(t, z)$ can be seen as a continuous function of $z$ into $\mathcal{D}^{\prime}(\mathbb{R})$. In the following Lemma we compute for every fixed $z$ the inverse cosine transform of this distribution. 
Lemma 6 Let $0 \leq v<d / 2$. For every $z \in \mathbb{R}^{d}, \mathcal{C}^{-1}\left(\partial .\left(E_{v}-\check{E}_{v}\right)(\cdot, z)\right)$ is a function and for all $t \in \mathbb{R}$

$$
\mathcal{C}^{-1}\left(\partial .\left(E_{v}-\check{E}_{v}\right)(\cdot, z)\right)(t)=\pi^{-d / 2} 2^{-v-d / 2}|t|^{-2 v-1+d} \frac{J_{-v+d / 2-1}(t|z|)}{(t|z|)^{-v+d / 2-1}} .
$$

Proof Since by Proposition 4 (i) and (iii)

$$
E_{v}(t, z)=2^{-2 v-1} \pi^{(1-d) / 2} \chi_{+}^{\nu+(1-d) / 2}\left(t^{2}-|z|^{2}\right) \quad t>0
$$

if $(d-1) / 2<v<d / 2$, then

$$
\partial_{t}\left(E_{v}-\check{E}_{v}\right)(t, z)
$$

is an even, locally integrable function in $t$, vanishing at $\infty$, so that its cosine transform is (see [7, Formula 11, Table 1.3, Chapter 1, page 12]),

$$
\begin{aligned}
\mathcal{C}^{-1} & \left(\partial .\left(E_{v}-\check{E}_{v}\right)(\cdot, z)\right)(s) \\
& =\frac{2}{\pi} \int_{0}^{+\infty} \partial_{t} E_{v}(t, z) \cos (s t) d t \\
& =\frac{2^{-2 v+1}}{\pi^{(d+1) / 2}} \frac{1}{\Gamma(v+(1-d) / 2)} \int_{|z|}^{+\infty} t\left(t^{2}-|z|^{2}\right)^{v+(1-d) / 2-1} \cos (s t) d t \\
& =\pi^{-d / 2} 2^{-v-d / 2}|s|^{-2 v-1+d} \frac{J_{-v+d / 2-1}(s|z|)}{(s|z|)^{-v+d / 2-1}} .
\end{aligned}
$$

Observe now that the distribution $\chi_{+}^{\nu+(1-d) / 2}$ in $\mathcal{D}^{\prime}(\mathbb{R})$ is entire in the variable $v$, and so is the distribution $\partial_{t}\left(E_{v}-\check{E}_{v}\right)(t, z)$ in $\mathcal{D}^{\prime}(\mathbb{R})$ for fixed $z$. This implies that also the cosine transform

$$
\mathcal{C}^{-1}\left(\partial .\left(E_{v}-\check{E}_{v}\right)(\cdot, z)\right)(s)
$$

can be analytically extended to all complex values of $v$ (see [9, Note 1, page 171]). This analytic extension coincides therefore with the analytic extension of the distribution

$$
\pi^{-d / 2} 2^{-v-d / 2}|s|^{-2 v-1+d} \frac{J_{-v+d / 2-1}(s|z|)}{(s|z|)^{-v+d / 2-1}} .
$$

Observe that this is the product of the locally integrable function $|s|^{-2 v-1+d}$ (recall that $v<d / 2)$ with the smooth function $\pi^{-d / 2} 2^{-v-d / 2} \frac{J_{-v+d / 2-1}(s|z|)}{(s|z|)^{-v+d / 2-1}}$, which is analytic in $v \in \mathbb{C}$.

Thus, the identity

$$
\mathcal{C}^{-1}\left(\partial .\left(E_{v}-\check{E}_{v}\right)(\cdot, z)\right)(s)=\pi^{-d / 2} 2^{-v-d / 2}|s|^{-2 v-1+d} \frac{J_{-v+d / 2-1}(s|z|)}{(s|z|)^{-v+d / 2-1}}
$$


holds for all $v<d / 2$.

\section{Proof of the main result}

It suffices to show the main inequality (2) for any positive integer $N$ and for any integer $X$ sufficiently large. Indeed, if $1 \leq X<X_{0}$

$$
\begin{aligned}
\sum_{m=0}^{X}\left|\sum_{j=1}^{N} a_{j} \varphi_{m}(x(j))\right|^{2} & \geq\left|\sum_{j=1}^{N} a_{j} \varphi_{0}(x(j))\right|^{2}=\left(\sum_{j=1}^{N} a_{j}\right)^{2} \\
& \geq \sum_{j=1}^{N} a_{j}^{2} \geq \frac{1}{X_{0}} X \sum_{j=1}^{N} a_{j}^{2} .
\end{aligned}
$$

Let $\kappa$ be a positive integer that we will choose later and let $Y=\kappa X$. By [10, Theorem 2], we can split the manifold $\mathcal{M}$ into $Y$ disjoint regions $\left\{\mathcal{R}_{i}\right\}_{i=1}^{Y}$ with measure $\left|\mathcal{R}_{i}\right|=1 / Y$ and such that each region contains a ball of radius $c_{1} Y^{-1 / d}$ and is contained in a ball of radius $c_{2} Y^{-1 / d}$, for appropriate values of $c_{1}$ and $c_{2}$ independent of $Y$. Let us call $\left\{\mathcal{B}_{r}\right\}_{r=1}^{R}$ the sequence of all the regions of the above collection $\left\{\mathcal{R}_{i}\right\}_{i=1}^{Y}$ which contain at least one of the points $x(j)$. We call $K_{r}$ the cardinality of the set $\left\{j=1, \ldots, N: x(j) \in \mathcal{B}_{r}\right\}$ and $S_{r}$ the sum of the weights $\left\{a_{j}\right\}$ corresponding to points $x(j) \in \mathcal{B}_{r}$. Without loss of generality we can assume that

$$
S_{1} \geq S_{2} \geq \ldots \geq S_{R}>0
$$

We rename the sequence $\{x(j)\}_{j=1}^{N}$ as

$$
\left\{x_{r, j}\right\}_{\substack{r=1, \ldots, R \\ j=1, \ldots, K_{r}}}
$$

with $x_{r, j} \in \mathcal{B}_{r}$ for all $j=1, \ldots, K_{r}$, and the sequence $\left\{a_{j}\right\}_{j=1}^{N}$ as

$$
\left\{a_{r, j}\right\}_{\substack{r=1, \ldots, R \\ j=1, \ldots, K_{r}}}
$$

Observe that

$$
S_{r}=\sum_{j=1}^{K_{r}} a_{r, j}
$$

Inequality (2) is an immediate consequence of the following

$$
\sum_{m=0}^{X}\left|\sum_{r=1}^{R} \sum_{j=1}^{K_{r}} a_{r, j} \varphi_{m}\left(x_{r, j}\right)\right|^{2} \geq C X \sum_{r=1}^{R}\left(\sum_{j=1}^{K_{r}} a_{r, j}\right)^{2} .
$$


Let $\psi$ be a smooth radial function on $\mathbb{R}^{d}$ compactly supported in the ball $B(0,1 / 2)=\left\{x \in \mathbb{R}^{d}:|x| \leqslant 1 / 2\right\}$ such that $\|\psi\|_{2}=1$ and $\int \psi>0$, and set $H(x)=\psi *_{d} \psi(x)$. Then clearly $H$ is radial, compactly supported in $B(0,1)$, $H(x) \leq 1$ for all $x \in \mathbb{R}^{d}$, and $H(0)=1$. Moreover its Fourier transform is $\mathcal{F}_{d} H(\xi)=\left(\mathcal{F}_{d} \psi(\xi)\right)^{2} \geq 0$ for all $\xi \in \mathbb{R}^{d}$, and has fast decay at infinity with all its derivatives.

If we now identify $H(x)$ with its radial profile, we can write

$$
\begin{aligned}
& \sum_{m=0}^{X}\left|\sum_{r=1}^{R} \sum_{j=1}^{K_{r}} a_{r, j} \varphi_{m}\left(x_{r, j}\right)\right|^{2} \\
& \quad \geq \sum_{m=0}^{+\infty} H\left(\frac{\lambda_{m}}{\lambda_{X}}\right)\left|\sum_{r=1}^{R} \sum_{j=1}^{K_{r}} a_{r, j} \varphi_{m}\left(x_{r, j}\right)\right|^{2} \\
& \quad=\sum_{r=1}^{R} \sum_{j=1}^{K_{r}} \sum_{s=1}^{R} \sum_{i=1}^{K_{s}} a_{r, j} a_{s, i}\left(\sum_{m=0}^{+\infty} H\left(\frac{\lambda_{m}}{\lambda_{X}}\right) \varphi_{m}\left(x_{r, j}\right) \overline{\varphi_{m}\left(x_{s, i}\right)}\right) .
\end{aligned}
$$

Let us define the kernel

$$
F_{X}(x, y):=\sum_{m=0}^{+\infty} H\left(\frac{\lambda_{m}}{\lambda_{X}}\right) \varphi_{m}(x) \overline{\varphi_{m}(y)}
$$

We will estimate $F_{X}(x, y)$ using the parametrix for the wave operator described in the previous section. For this, one would need that the Fourier cosine transform of $H\left(\frac{\dot{\partial}}{\lambda_{X}}\right)$ have small support. This of course cannot be achieved, having $H$ itself compact support. For this reason we pick $\eta=\mathcal{F}_{d} \phi$ where $\phi(\xi)$ is a nonnegative smooth radial function supported in $B(0, \varepsilon / 2 \pi)$ and such that $\phi(\xi)=1$ in $B(0, \varepsilon / 4 \pi)$ and define

$$
\widetilde{H}(x)=H\left(\frac{\cdot}{\lambda_{X}}\right) *_{d} \eta(x) .
$$

The reason for taking a $d$-dimensional convolution will be clarified in Lemma 8 where we use the fact that $\mathcal{F}_{d} \widetilde{H} \geq 0$.

Observe that supp $\mathcal{F}_{d} \widetilde{H} \subset B(0, \varepsilon / 2 \pi)$. It is remarkable that the cosine transform of $\widetilde{H}$ has support in $[0, \varepsilon]$ and is nonnegative.

Lemma $7 \mathcal{C}^{-1} \widetilde{H}(\rho) \geq 0$ for $\rho \geq 0$ and $\mathcal{C}^{-1} \widetilde{H}(\rho)=0$ for $\rho>\varepsilon$.

Proof It is known (see [19, eq. (3.9)] ) that for $d>d^{\prime} \geq 1$,

$$
\mathcal{F}_{d^{\prime}}\left(\mathcal{F}_{d} g\right)(s)=c_{d, d^{\prime}} \int_{s}^{+\infty}\left(r^{2}-s^{2}\right)^{\left(d-d^{\prime}\right) / 2-1} r g(r) d r
$$


Let now $g(r)=\mathcal{F}_{d} \widetilde{H}(r)$. Since $\widetilde{H}(s)=\mathcal{F}_{d} g(s)$ and the cosine transform is essentially $\mathcal{F}_{1}$ we obtain

$$
\mathcal{C}^{-1} \tilde{H}(\rho)=\frac{1}{\pi} \mathcal{F}_{1} \mathcal{F}_{d} g\left(\frac{\rho}{2 \pi}\right)
$$

and the thesis follows immediately from (13), the fact that $g(r) \geq 0$ and the fact that $g(r)=0$ for $r>\varepsilon / 2 \pi$.

Let us go back to the kernel $F_{X}$,

$$
\begin{aligned}
F_{X}(x, y) & =\sum_{m=0}^{+\infty} H\left(\frac{\lambda_{m}}{\lambda_{X}}\right) \varphi_{m}(x) \overline{\varphi_{m}(y)} \\
& =\sum_{m=0}^{+\infty} \widetilde{H}\left(\lambda_{m}\right) \varphi_{m}(x) \overline{\varphi_{m}(y)}+\sum_{m=0}^{+\infty}\left(H\left(\frac{\lambda_{m}}{\lambda_{X}}\right)-\widetilde{H}\left(\lambda_{m}\right)\right) \varphi_{m}(x) \overline{\varphi_{m}(y)}
\end{aligned}
$$

Since

$$
\widetilde{H}(\lambda)=\int_{0}^{+\infty} \mathcal{C}^{-1} \tilde{H}(t) \cos (\lambda t) d t
$$

and $\mathcal{C}^{-1} \widetilde{H}(t)$ is supported in $[-\varepsilon, \varepsilon]$, by Theorem 5 , we can write

$$
\begin{aligned}
& \sum_{m=0}^{+\infty} \tilde{H}\left(\lambda_{m}\right) \varphi_{m}(x) \overline{\varphi_{m}(y)} \\
& \quad=\sum_{m=0}^{+\infty} \int_{0}^{+\infty} \mathcal{C}^{-1} \widetilde{H}(t) \cos \left(\lambda_{m} t\right) d t \varphi_{m}(x) \overline{\varphi_{m}(y)} \\
& \quad=\frac{1}{2} \sum_{m=0}^{+\infty}\left\langle\cos \left(\lambda_{m} \cdot\right) \varphi_{m}(x) \overline{\varphi_{m}(y)}, \mathcal{C}^{-1} \widetilde{H}\right\rangle \\
& =\frac{1}{2}\left\langle\cos (\cdot \sqrt{\Delta})(x, y), \mathcal{C}^{-1} \widetilde{H}\right\rangle \\
& \quad=\frac{1}{2} \sum_{\nu=0}^{Q} \alpha_{v}(x, y) \Omega_{v}(x, y)+\int_{0}^{\varepsilon} R_{Q}(t, x, y) \mathcal{C}^{-1} \tilde{H}(t) d t
\end{aligned}
$$

where we set

$$
\Omega_{v}(x, y)=\left\langle\partial_{t}\left(E_{v}-\check{E}_{v}\right)(\cdot, d(x, y)), \mathcal{C}^{-1} \widetilde{H}\right\rangle .
$$

We can therefore decompose the kernel $F_{X}$ as follows

$$
F_{X}(x, y)=\sum_{n=1}^{5} F_{n}(x, y),
$$


where

$$
\begin{aligned}
& F_{1}(x, y)=\frac{1}{2} \alpha_{0}(x, y) \Omega_{0}(x, y), \\
& F_{2}(x, y)=\frac{1}{2} \sum_{1 \leq v<d / 2} \alpha_{v}(x, y) \Omega_{v}(x, y), \\
& F_{3}(x, y)=\frac{1}{2} \sum_{d / 2 \leq v \leq Q} \alpha_{v}(x, y) \Omega_{v}(x, y), \\
& F_{4}(x, y)=\int_{0}^{\varepsilon} R_{Q}(t, x, y) \mathcal{C}^{-1} \widetilde{H}(t) d t, \\
& F_{5}(x, y)=\sum_{m=0}^{+\infty}\left(H\left(\frac{\lambda_{m}}{\lambda_{X}}\right)-\widetilde{H}\left(\lambda_{m}\right)\right) \varphi_{m}(x) \overline{\varphi_{m}(y) .}
\end{aligned}
$$

Recalling (11) and (12) we have

$$
\begin{aligned}
\sum_{m=0}^{X}\left|\sum_{r=1}^{R} \sum_{j=1}^{K_{r}} a_{r, j} \varphi_{m}\left(x_{r, j}\right)\right|^{2} & =\sum_{r=1}^{R} \sum_{j=1}^{K_{r}} \sum_{s=1}^{R} \sum_{i=1}^{K_{s}} a_{r, j} a_{s, i} F_{X}\left(x_{r, j}, x_{s, i}\right) \\
& =\sum_{n=1}^{5} \sum_{r=1}^{R} \sum_{j=1}^{K_{r}} \sum_{s=1}^{R} \sum_{i=1}^{K_{s}} a_{r, j} a_{s, i} F_{n}\left(x_{r, j}, x_{s, i}\right) .
\end{aligned}
$$

We start estimating the term with $F_{1}$ which is the positive part of the kernel and gives the main contribution.

Lemma 8 For $\kappa$ large enough there exist $X_{0}>0$ and $C>0$ such that for every $X>X_{0}$

$$
\sum_{r=1}^{R} \sum_{j=1}^{K_{r}} \sum_{s=1}^{R} \sum_{i=1}^{K_{s}} a_{r, j} a_{s, i} F_{1}\left(x_{r, j}, x_{s, i}\right) \geq C X \sum_{r=1}^{R}\left(\sum_{j=1}^{K_{r}} a_{r, j}\right)^{2}
$$

Proof First of all we show that $\Omega_{0}(x, y)$ is positive. Indeed, by Lemma 6 and (8), for every $x, y \in \mathcal{M}$,

$$
\begin{aligned}
\Omega_{0}(x, y) & =\left\langle\mathcal{C}^{-1}\left(\partial_{t}\left(E_{0}-\check{E}_{0}\right)(\cdot, d(x, y))\right), \widetilde{H}\right\rangle \\
& =2 \int_{0}^{+\infty} \mathcal{C}^{-1}\left(\partial_{t}\left(E_{0}-\check{E}_{0}\right)(\cdot, d(x, y))\right)(t) \widetilde{H}(t) d t \\
& =\frac{2}{d(x, y)^{d / 2-1}(2 \pi)^{d / 2}} \int_{0}^{+\infty} J_{d / 2-1}\left(2 \pi \frac{d(x, y)}{2 \pi} t\right) \widetilde{H}(t) t^{d / 2} d t
\end{aligned}
$$




$$
\begin{aligned}
& =\frac{2}{(2 \pi)^{d}} \mathcal{F}_{d} \widetilde{H}\left(\frac{d(x, y)}{2 \pi}\right) \\
& =\frac{2}{(2 \pi)^{d}} \lambda_{X}^{d} \mathcal{F}_{d} H\left(\frac{\lambda_{X} d(x, y)}{2 \pi}\right) \mathcal{F}_{d} \eta\left(\frac{d(x, y)}{2 \pi}\right) \geq 0 .
\end{aligned}
$$

Since also $\alpha_{0}(x, y)$ is positive, we can disregard off-diagonal terms,

$$
\begin{aligned}
& \sum_{r=1}^{R} \sum_{j=1}^{K_{r}} \sum_{s=1}^{R} \sum_{i=1}^{K_{s}} a_{r, j} a_{s, i} F_{1}\left(x_{r, j}, x_{s, i}\right) \\
& \quad=\frac{1}{2} \sum_{r=1}^{R} \sum_{j=1}^{K_{r}} \sum_{s=1}^{R} \sum_{i=1}^{K_{s}} a_{r, j} a_{s, i} \alpha_{0}\left(x_{r, j}, x_{s, i}\right) \Omega_{0}\left(x_{r, j}, x_{s, i}\right) \\
& \quad \geqslant \frac{1}{2} \sum_{r=1}^{R} \sum_{j=1}^{K_{r}} \sum_{i=1}^{K_{r}} a_{r, j} a_{r, i} \alpha_{0}\left(x_{r, j}, x_{r, i}\right) \Omega_{0}\left(x_{r, j}, x_{r, i}\right) \\
& \quad=\frac{\lambda_{X}^{d}}{(2 \pi)^{d}} \sum_{r=1}^{R} \sum_{j=1}^{K_{r}} \sum_{i=1}^{K_{r}} a_{r, j} a_{r, i} \alpha_{0}\left(x_{r, j}, x_{r, i}\right) \mathcal{F}_{d} H\left(\frac{\lambda_{X} d\left(x_{r, j}, x_{r, i}\right)}{2 \pi}\right) \mathcal{F}_{d \eta} \eta\left(\frac{d\left(x_{r, j}, x_{r, i}\right)}{2 \pi}\right) .
\end{aligned}
$$

By Weyl's estimate (see e.g. [11, III, Corollary 17.5.8]) $\lambda_{X} \sim X^{1 / d}$. Thus, if $x, y \in \mathcal{B}_{r}$ then

$$
\lambda_{X} d(x, y) \leq \lambda_{X} 2 c_{2}(\kappa X)^{-1 / d} \leq c_{3} \kappa^{-1 / d}
$$

Let $\kappa$ large enough so that if $x, y \in \mathcal{B}_{r}$

$$
\mathcal{F}_{d} H\left(\frac{\lambda_{X} d(x, y)}{2 \pi}\right)=\left(\mathcal{F}_{d} \psi\right)^{2}\left(\frac{\lambda_{X} d(x, y)}{2 \pi}\right) \geq \frac{\left(\mathcal{F}_{d} \psi\right)^{2}(0)}{2}>0
$$

and

$$
\frac{d(x, y)}{2 \pi} \leq \frac{\varepsilon}{4 \pi},
$$

so that

$$
\mathcal{F}_{d} \eta\left(\frac{d(x, y)}{2 \pi}\right)=1
$$


Finally,

$$
\begin{aligned}
& \sum_{r=1}^{R} \sum_{j=1}^{K_{r}} \sum_{s=1}^{R} \sum_{i=1}^{K_{s}} a_{r, j} a_{s, i} F_{1}\left(x_{r, j}, x_{s, i}\right) \\
& \quad \geq C X \sum_{r=1}^{R} \sum_{j=1}^{K_{r}} \sum_{i=1}^{K_{r}} a_{r, j} a_{r, i}=C X \sum_{r=1}^{R}\left(\sum_{j=1}^{K_{r}} a_{r, j}\right)^{2} .
\end{aligned}
$$

The following lemmas show that the contributions given by the terms with $F_{2}, F_{3}$, $F_{4}, F_{5}$ are negligible.

Lemma 9 There exist $C>0$ and $X_{0}>0$ such that for every $X>X_{0}$

$$
\left|\sum_{r=1}^{R} \sum_{j=1}^{K_{r}} \sum_{s=1}^{R} \sum_{i=1}^{K_{s}} a_{r, j} a_{s, i} F_{2}\left(x_{r, j}, x_{s, i}\right)\right| \leq C X^{1-2 / d} \sum_{r=1}^{R}\left(\sum_{j=1}^{K_{r}} a_{r, j}\right)^{2} .
$$

Proof We will show that for every integer $v, 1 \leq v<d / 2$,

$$
\left|\sum_{r=1}^{R} \sum_{j=1}^{K_{r}} \sum_{s=1}^{R} \sum_{i=1}^{K_{s}} a_{r, j} a_{s, i} \alpha_{v}\left(x_{r, j}, x_{s, i}\right) \Omega_{v}\left(x_{r, j}, x_{s, i}\right)\right| \leq C X^{1-2 v / d} \sum_{r=1}^{R}\left(\sum_{j=1}^{K_{r}} a_{r, j}\right)^{2} .
$$

By Lemma 6, for every $x, y \in \mathcal{M}$,

$$
\begin{aligned}
\Omega_{v}(x, y) & =\left\langle\mathcal{C}^{-1}\left(\partial .\left(E_{v}-\check{E}_{v}\right)(\cdot, d(x, y))\right), \widetilde{H}\right\rangle \\
& =2 \int_{0}^{+\infty} \pi^{-d / 2} 2^{-v-d / 2}|t|^{-2 v-1+d} \frac{J_{-v+d / 2-1}(d(x, y) t)}{(d(x, y) t)^{-v+d / 2-1}} \widetilde{H}(t) d t \\
& =\pi^{\nu-d} 2^{1-d} \mathcal{F}_{d-2 v} \widetilde{H}\left(\frac{d(x, y)}{2 \pi}\right) \\
& =\pi^{\nu-d} 2^{1-d} \mathcal{F}_{d-2 v}\left(H\left(\frac{\cdot}{\lambda_{X}}\right) * d \eta\right)\left(\frac{d(x, y)}{2 \pi}\right) .
\end{aligned}
$$

Using (13) and the fast decay at infinity of $\mathcal{F}_{d} \psi$, for any positive $M$ there exist positive constants $C$ and $G$ such that for every $\rho \geq 0$

$$
\begin{aligned}
& \left|\mathcal{F}_{d-2 v}\left(H\left(\frac{\cdot}{\lambda_{X}}\right) *_{d} \eta\right)(\rho)\right|=\lambda_{X}^{d}\left|\mathcal{F}_{d-2 v}\left(\mathcal{F}_{d}\left(\left(\mathcal{F}_{d} \psi\right)^{2}\left(\lambda_{X} \cdot\right) \mathcal{F}_{d} \eta\right)\right)(\rho)\right| \\
& =c_{d, d-2 v} \lambda_{X}^{d} \int_{\rho}^{+\infty}\left(r^{2}-\rho^{2}\right)^{\nu-1} r\left(\mathcal{F}_{d} \psi\left(\lambda_{X} r\right)\right)^{2} \mathcal{F}_{d} \eta(r) d r
\end{aligned}
$$




$$
\begin{aligned}
& \leq C \lambda_{X}^{d} \int_{\rho}^{+\infty}\left(r^{2}-\rho^{2}\right)^{\nu-1} r\left(\mathcal{F}_{d} \psi\left(\lambda_{X} r\right)\right)^{2} d r \\
& \leq C \lambda_{X}^{d} \int_{\rho}^{+\infty}\left(r^{2}-\rho^{2}\right)^{\nu-1} r \frac{C}{\left(1+\lambda_{X} r\right)^{G}} d r \\
& \leq C \lambda_{X}^{d-2 v} \int_{\lambda_{X} \rho}^{+\infty}\left(u^{2}-\left(\lambda_{X} \rho\right)^{2}\right)^{\nu-1} u \frac{C}{(1+u)^{G}} d u \leq \frac{C \lambda_{X}^{d-2 v}}{\left(1+\left|\lambda_{X} \rho\right|\right)^{M}}
\end{aligned}
$$

Therefore, using the symmetry of $\Omega_{v}(x, y)$, for any integer $v$ with $1 \leq v<d / 2$ we obtain

$$
\begin{aligned}
& \left|\sum_{r=1}^{R} \sum_{j=1}^{K_{r}} \sum_{s=1}^{R} \sum_{i=1}^{K_{s}} a_{r, j} a_{s, i} \alpha_{v}\left(x_{r, j}, x_{s, i}\right) \Omega_{v}\left(x_{r, j}, x_{s, i}\right)\right| \\
& \quad \leq 2 C \sum_{r=1}^{R} \sum_{j=1}^{K_{r}} \sum_{s=r}^{R} \sum_{i=1}^{K_{s}} a_{r, j} a_{s, i}\left|\Omega_{v}\left(x_{r, j}, x_{s, i}\right)\right| \\
& \quad \leq C \sum_{r=1}^{R} \sum_{j=1}^{K_{r}} \sum_{s=r}^{R} \sum_{i=1}^{K_{s}} a_{r, j} a_{s, i} \lambda_{X}^{d-2 v} \frac{1}{\left(1+\lambda_{X} d\left(x_{r, j}, x_{s, i}\right)\right)^{M}} .
\end{aligned}
$$

In order to estimate the above sum recall that every region $\mathcal{B}_{r}$ is contained in a ball centered at a point $z_{r} \in \mathcal{B}_{r}$ of radius $c_{2} Y^{-1 / d}$ and let $c_{3}=10 c_{2}$. For every fixed $r=1, \ldots, R$ we will consider separately the contribution of those values of $s$ for which the $\mathcal{B}_{S}$ is near $\mathcal{B}_{r}$, in the sense that $\mathcal{B}_{s}$ is contained in the ball centered at $z_{r}$ and with radius $c_{3} Y^{-1 / d}$, and the contribution of the remaining values of $s$, for which we will say that $\mathcal{B}_{s}$ is far from $\mathcal{B}_{r}$. Notice that there are at most

$$
\frac{\left|B\left(z_{r}, c_{3} Y^{-1 / d}\right)\right|}{Y^{-1}} \leq \frac{C\left(c_{3} Y^{-1 / d}\right)^{d}}{Y^{-1}} \leq C c_{3}^{d}
$$

regions $\mathcal{B}_{s}$ near $\mathcal{B}_{r}$. Thus, using again that $\lambda_{X} \sim X^{1 / d}$ and that for $r \leqslant s$ we have $\sum_{j=1}^{K_{r}} a_{r, j} \geq \sum_{i=1}^{K_{s}} a_{s, i}$, we obtain

$$
\begin{aligned}
& \sum_{r=1}^{R} \sum_{j=1}^{K_{r}} \sum_{s=r}^{R} \sum_{i=1}^{K_{s}} a_{r, j} a_{s, i} \lambda_{X}^{d-2 v} \frac{1}{\left(1+\lambda_{X} d\left(x_{r, j}, x_{s, i}\right)\right)^{M}} \\
& \leq C X^{1-2 v / d} \sum_{r=1}^{R} \sum_{\substack{s=r \\
B_{s} \text { near } B_{r}}}^{R} \sum_{j=1}^{K_{r}} a_{r, j} \sum_{i=1}^{K_{s}} a_{s, i} \\
& \quad+C X^{1-2 v / d} \sum_{r=1}^{R} \sum_{\substack{s=r \\
B_{s} \text { far from } B_{r}}}^{R} \sum_{j=1}^{K_{r}} a_{r, j} \sum_{i=1}^{K_{s}} a_{s, i}\left(\lambda_{X} d\left(x_{r, j}, x_{s, i}\right)\right)^{-M}
\end{aligned}
$$




$$
\begin{aligned}
\leq & C X^{1-2 v / d} \sum_{r=1}^{R}\left(\sum_{j=1}^{K_{r}} a_{r, j}\right)^{2} \\
& +C X^{1-2 v / d} \sum_{r=1}^{R-1} \sum_{\substack{s=r+1 \\
B_{s} \text { far from } B_{r}}}^{R} \sum_{j=1}^{K_{r}} a_{r, j} \sum_{i=1}^{K_{s}} a_{s, i}\left(X^{1 / d} d\left(x_{r, j}, x_{s, i}\right)\right)^{-M} .
\end{aligned}
$$

Using again that for $r \leq s$ we have $\sum_{j=1}^{K_{r}} a_{r, j} \geq \sum_{i=1}^{K_{s}} a_{s, i}$,

$$
\begin{aligned}
& \sum_{r=1}^{R-1} \sum_{\substack{s=r+1 \\
B_{s} \text { far from } B_{r}}}^{R} \sum_{j=1}^{K_{r}} a_{r, j} \sum_{i=1}^{K_{s}} a_{s, i}\left(X^{1 / d} d\left(x_{r, j}, x_{s, i}\right)\right)^{-M} \\
& =\sum_{r=1}^{R-1} \sum_{j=1}^{K_{r}} a_{r, j} \sum_{\ell=0}^{\infty} \sum_{2^{\ell-1} c_{3} Y^{-1 / d} \leq d\left(z_{r}, z_{s}\right) \leq 2^{\ell} c_{3} Y^{-1 / d}} \sum_{i=1}^{K_{s}} a_{s, i}\left(X^{1 / d} d\left(x_{r, j}, x_{s, i}\right)\right)^{-M} \\
& \leq C \sum_{r=1}^{R-1} \sum_{j=1}^{K_{r}} a_{r, j} \sum_{\ell=0}^{\infty} 2^{-\ell M} \sum_{s>r:} \sum_{i=1}^{K_{s}} a_{s, i} \\
& \leq C \sum_{r=1}^{R-1} \sum_{j=1}^{K_{r}} a_{r, j} \sum_{\ell=0}^{\infty} 2^{-\ell M} \frac{\left(2^{\ell} Y^{-1 / d}\right)^{d}}{Y^{-1}} \sum_{j=1}^{K_{r}} a_{r, j} \\
& \leq C \sum_{r=1}^{R-1}\left(\sum_{j=1}^{K_{r}} a_{r, j}\right)^{2} \sum_{\ell=0}^{\infty} 2^{-\ell(M-d)} \leq C \sum_{r=1}^{R-1}\left(\sum_{j=1}^{K_{r}} a_{r, j}\right)^{2} .
\end{aligned}
$$

Lemma 10 There exist $C>0$ and $X_{0}$ such that for every $X>X_{0}$

$$
\left|\sum_{r=1}^{R} \sum_{j=1}^{K_{r}} \sum_{s=1}^{R} \sum_{i=1}^{K_{s}} a_{r, j} a_{s, i} F_{3}\left(x_{r, j}, x_{s, i}\right)\right| \leq C \sum_{r=1}^{R}\left(\sum_{j=1}^{K_{r}} a_{r, j}\right)^{2} .
$$

Proof We will show that for every integer $v \geq d / 2$,

$$
\begin{aligned}
& \left|\sum_{r=1}^{R} \sum_{j=1}^{K_{r}} \sum_{s=1}^{R} \sum_{i=1}^{K_{s}} a_{r, j} a_{s, i} \alpha_{v}\left(x_{r, j}, x_{s, i}\right) \Omega_{v}\left(x_{r, j}, x_{s, i}\right)\right| \\
& \quad \leq C X^{1-2 v / d} \sum_{r=1}^{R}\left(\sum_{j=1}^{K_{r}} a_{r, j}\right)^{2} .
\end{aligned}
$$


Observe that for $v \geq d / 2$, the distribution $\partial_{t}\left(E_{v}-\check{E}_{v}\right)(t, d(x, y))$ can be identified with the locally integrable function

$$
C_{v}|t|\left(t^{2}-d(x, y)^{2}\right)_{+}^{\nu-1+(1-d) / 2},
$$

for an appropriate value of $C_{v}$. Therefore, using the symmetry of $\Omega_{v}(x, y)$,

$$
\begin{aligned}
& \left|\sum_{r=1}^{R} \sum_{j=1}^{K_{r}} \sum_{s=1}^{R} \sum_{i=1}^{K_{s}} a_{r, j} a_{s, i} \alpha_{\nu}\left(x_{r, j}, x_{s, i}\right) \Omega_{v}\left(x_{r, j}, x_{s, i}\right)\right| \\
& \quad \leq C \sum_{r=1}^{R} \sum_{j=1}^{K_{r}} \sum_{s=r}^{R} \sum_{i=1}^{K_{s}} a_{r, j} a_{s, i} \int_{d\left(x_{r, j}, x_{s, i}\right)}^{+\infty} t\left(t^{2}-d\left(x_{r, j}, x_{s, i}\right)^{2}\right)^{\nu-1+(1-d) / 2} \mathcal{C}^{-1} \widetilde{H}(t) d t,
\end{aligned}
$$

where we use the fact that $\mathcal{C}^{-1} \tilde{H}(t) \geq 0$, by Lemma 7 .

Assume first that $d=1$ and let $D=d\left(x_{r, j}, x_{s, i}\right)$, then

$$
\begin{aligned}
& \int_{D}^{+\infty} t\left(t^{2}-D^{2}\right)^{\nu-1+(1-d) / 2} \mathcal{C}^{-1} \widetilde{H}(t) d t \\
& \quad=\int_{D}^{+\infty} t\left(t^{2}-D^{2}\right)^{\nu-1} \frac{1}{\pi} \mathcal{F}_{1} \widetilde{H}\left(\frac{t}{2 \pi}\right) d t \\
& \quad=\lambda_{X} \int_{D}^{+\infty} t\left(t^{2}-D^{2}\right)^{\nu-1} \frac{1}{\pi}\left(\mathcal{F}_{1} \psi\left(\lambda_{X} \frac{t}{2 \pi}\right)\right)^{2} \mathcal{F}_{1} \eta\left(\frac{t}{2 \pi}\right) d t \\
& \quad \leq c \lambda_{X} \int_{\frac{D}{2 \pi}}^{+\infty} u^{v}\left(u-\frac{D}{2 \pi}\right)^{v-1}\left(\mathcal{F}_{1} \psi\left(\lambda_{X} u\right)\right)^{2} \mathcal{F}_{1} \eta(u) d u
\end{aligned}
$$

A similar estimate can be obtained for $d \geq 2$. Indeed, by formula (13)

$$
\begin{aligned}
\mathcal{C}^{-1} \widetilde{H}(t) & =\frac{2}{\pi} \mathcal{C} \widetilde{H}(t) \\
& =\frac{1}{\pi} \mathcal{F}_{1} \mathcal{F}_{d} \mathcal{F}_{d} \widetilde{H}\left(\frac{t}{2 \pi}\right) \\
& =c \int_{\frac{t}{2 \pi}}^{+\infty}\left(u^{2}-\left(\frac{t}{2 \pi}\right)^{2}\right)^{(d-1) / 2-1} u \mathcal{F}_{d} \widetilde{H}(u) d u \\
& =c \lambda_{X}^{d} \int_{\frac{t}{2 \pi}}^{+\infty}\left(u^{2}-\left(\frac{t}{2 \pi}\right)^{2}\right)^{(d-1) / 2-1} u\left(\mathcal{F}_{d} \psi\left(\lambda_{X} u\right)\right)^{2} \mathcal{F}_{d} \eta(u) d u,
\end{aligned}
$$


so that, by Fubini's theorem,

$$
\begin{aligned}
& \int_{D}^{+\infty} t\left(t^{2}-D^{2}\right)^{\nu-1+(1-d) / 2} \mathcal{C}^{-1} \tilde{H}(t) d t \\
& =c \lambda_{X}^{d} \int_{\frac{D}{2 \pi}}^{+\infty} u\left(\mathcal{F}_{d} \psi\left(\lambda_{X} u\right)\right)^{2} \mathcal{F}_{d} \eta(u) \\
& \quad \times\left(\int_{D}^{2 \pi u} 2 t\left(t^{2}-D^{2}\right)^{\nu-d / 2-1 / 2}\left(u^{2}-\left(\frac{t}{2 \pi}\right)^{2}\right)^{(d-3) / 2} d t\right) d u
\end{aligned}
$$

Since

$$
\begin{aligned}
& \int_{D}^{U} t\left(t^{2}-D^{2}\right)^{\nu-d / 2-1 / 2}\left(U^{2}-t^{2}\right)^{(d-3) / 2} d t \\
& \leq C U^{(d-3) / 2} \int_{D}^{U} t^{\nu-d / 2+1 / 2}(t-D)^{\nu-d / 2-1 / 2}(U-t)^{(d-3) / 2} d t \\
& \leq C U^{(d-3) / 2} U^{\nu-d / 2+1 / 2} \\
& \quad \times \int_{0}^{1}(z(U-D))^{\nu-d / 2-1 / 2}((1-z)(U-D))^{(d-3) / 2}(U-D) d z \\
& =C U^{\nu-1}(U-D)^{\nu-1} \int_{0}^{1} z^{\nu-d / 2-1 / 2}(1-z)^{(d-3) / 2} d z,
\end{aligned}
$$

we obtain

$$
\begin{aligned}
& \int_{D}^{+\infty} t\left(t^{2}-D^{2}\right)^{\nu-1+(1-d) / 2} \mathcal{C}^{-1} \tilde{H}(t) d t \\
& \quad \leq c \lambda_{X}^{d} \int_{\frac{D}{2 \pi}}^{+\infty} u^{\nu}\left(u-\frac{D}{2 \pi}\right)^{\nu-1}\left(\mathcal{F}_{d} \psi\left(\lambda_{X} u\right)\right)^{2} \mathcal{F}_{d} \eta(u) d u
\end{aligned}
$$

Thus, for all $d \geq 1$,

$$
\begin{aligned}
& \int_{D}^{+\infty} t\left(t^{2}-D^{2}\right)^{v-1+(1-d) / 2} \mathcal{C}^{-1} \widetilde{H}(t) d t \\
& \quad \leq c \lambda_{X}^{d} \int_{\frac{D}{2 \pi}}^{+\infty} u^{v}\left(u-\frac{D}{2 \pi}\right)^{v-1}\left(\mathcal{F}_{d} \psi\left(\lambda_{X} u\right)\right)^{2} \mathcal{F}_{d} \eta(u) d u \\
& \quad \leq c \lambda_{X}^{d-1} \int_{\lambda_{X} \frac{D}{2 \pi}}^{+\infty}\left(\frac{v}{\lambda_{X}}\right)^{v}\left(\frac{v}{\lambda_{X}}-\frac{D}{2 \pi}\right)^{v-1}\left(\mathcal{F}_{d} \psi(v)\right)^{2} \mathcal{F}_{d} \eta\left(\frac{v}{\lambda_{x}}\right) d v \\
& \quad \leq c \lambda_{X}^{d-2 v} \int_{\lambda_{X} \frac{D}{2 \pi}}^{+\infty} v^{v}\left(v-\frac{D \lambda_{X}}{2 \pi}\right)^{v-1}\left(\mathcal{F}_{d} \psi(v)\right)^{2} d v
\end{aligned}
$$




$$
\begin{aligned}
& \leq c \lambda_{X}^{d-2 v} \int_{\lambda_{X} \frac{D}{2 \pi}}^{+\infty} v^{2 v-1}\left(\mathcal{F}_{d} \psi(v)\right)^{2} d v \\
& \leq c \lambda_{X}^{d-2 v} \frac{1}{\left(1+\lambda_{X} D\right)^{M}} .
\end{aligned}
$$

Finally, arguing as in the previous lemma,

$$
\begin{aligned}
& \sum_{r=1}^{R} \sum_{j=1}^{K_{r}} \sum_{s=r}^{R} \sum_{i=1}^{K_{s}} a_{r, j} a_{s, i} \int_{d\left(x_{r, j}, x_{s, i}\right)}^{+\infty} t\left(t^{2}-d\left(x_{r, j}, x_{s, i}\right)^{2}\right)^{\nu-1+(1-d) / 2} \mathcal{C}^{-1} \widetilde{H}(t) d t \\
& \quad \leq C \sum_{r=1}^{R} \sum_{j=1}^{K_{r}} \sum_{s=r}^{R} \sum_{i=1}^{K_{s}} a_{r, j} a_{s, i} \lambda_{X}^{d-2 v} \frac{1}{\left(1+\lambda_{X} d\left(x_{r, j}, x_{s, i}\right)\right)^{M}} \\
& \quad \leq C X^{1-2 v / d} \sum_{r=1}^{R}\left(\sum_{j=1}^{K_{r}} a_{r, j}\right)^{2} .
\end{aligned}
$$

Lemma 11 There exist $C>0$ and $X_{0}>0$ such that for every $X>X_{0}$

$$
\left|\sum_{r=1}^{R} \sum_{j=1}^{K_{r}} \sum_{s=1}^{R} \sum_{i=1}^{K_{s}} a_{r, j} a_{s, i} F_{4}\left(x_{r, j}, x_{s, i}\right)\right| \leq C \sum_{r=1}^{R}\left(\sum_{j=1}^{K_{r}} a_{r, j}\right)^{2} .
$$

Proof Recall that for every $x, y \in \mathcal{M}$,

$$
F_{4}(x, y)=\int_{0}^{\varepsilon} R_{Q}(t, x, y) \mathcal{C}^{-1} \widetilde{H}(t) d t .
$$

As before, if $d=1$, then

$$
\mathcal{C}^{-1} \widetilde{H}(t)=\frac{1}{\pi} \mathcal{F}_{1} \tilde{H}\left(\frac{t}{2 \pi}\right)=\frac{\lambda_{X}}{\pi}\left(\mathcal{F}_{1} \psi\left(\lambda_{X} \frac{t}{2 \pi}\right)\right)^{2} \mathcal{F}_{1} \eta\left(\frac{t}{2 \pi}\right)
$$

and, by Theorem 5 ,

$$
\begin{aligned}
\left|F_{4}(x, y)\right| & \leq \frac{\lambda_{X}}{\pi} \int_{0}^{\varepsilon}\left|R_{Q}(t, x, y)\right|\left(\mathcal{F}_{1} \psi\left(\lambda_{X} \frac{t}{2 \pi}\right)\right)^{2} \mathcal{F}_{1} \eta\left(\frac{t}{2 \pi}\right) d t \\
& \leq c \lambda_{X}^{1-2 Q-2} \int_{0}^{+\infty} t^{2 Q+1}\left(\mathcal{F}_{1} \psi(t)\right)^{2} d t
\end{aligned}
$$

A similar estimate holds for $d \geq 2$. Indeed, as in the previous lemma,

$$
\mathcal{C}^{-1} \tilde{H}(t)=c \lambda_{X}^{d} \int_{\frac{t}{2 \pi}}^{+\infty}\left(u^{2}-\left(\frac{t}{2 \pi}\right)^{2}\right)^{(d-1) / 2-1} u\left(\mathcal{F}_{d} \psi\left(\lambda_{X} u\right)\right)^{2} \mathcal{F}_{d} \eta(u) d u
$$


so that, again by Theorem 5 ,

$$
\begin{aligned}
& \left|F_{4}(x, y)\right| \\
& =c\left|\lambda_{X}^{d} \int_{0}^{\varepsilon} R_{Q}(t, x, y) \int_{\frac{t}{2 \pi}}^{+\infty}\left(u^{2}-\left(\frac{t}{2 \pi}\right)^{2}\right)^{(d-1) / 2-1} u\left(\mathcal{F}_{d} \psi\left(\lambda_{X} u\right)\right)^{2} \mathcal{F}_{d} \eta(u) d u d t\right| \\
& \leq c \lambda_{X}^{d} \int_{0}^{+\infty} u\left(\mathcal{F}_{d} \psi\left(\lambda_{X} u\right)\right)^{2} \mathcal{F}_{d} \eta(u) \\
& \quad \times \int_{0}^{\min (2 \pi u, \varepsilon)}\left|R_{Q}(t, x, y)\right|\left(u^{2}-\left(\frac{t}{2 \pi}\right)^{2}\right)^{(d-1) / 2-1} d t d u \\
& \leq c \lambda_{X}^{d} \int_{0}^{+\infty} u\left(\mathcal{F}_{d} \psi\left(\lambda_{X} u\right)\right)^{2} \mathcal{F}_{d} \eta(u) \int_{0}^{2 \pi u} t^{2 Q+2-d}\left(u^{2}-\left(\frac{t}{2 \pi}\right)^{2}\right)^{(d-1) / 2-1} d t d u \\
& \leq c \lambda_{X}^{d} \int_{0}^{+\infty}\left(\mathcal{F}_{d} \psi\left(\lambda_{X} u\right)\right)^{2} \mathcal{F}_{d} \eta(u) u^{2 Q+1} d u . \\
& \leq c \lambda_{X}^{d-2 Q-2} \int_{0}^{+\infty}\left(\mathcal{F}_{d} \psi(v)\right)^{2} v^{2 Q+1} d v .
\end{aligned}
$$

Finally, for all $d \geq 1$, since $R \leq Y=\kappa X$,

$$
\begin{aligned}
& \left|\sum_{r=1}^{R} \sum_{j=1}^{K_{r}} \sum_{s=1}^{R} \sum_{i=1}^{K_{s}} a_{r, j} a_{s, i} \int_{0}^{\varepsilon} R_{Q}\left(t, x_{r, j}, x_{s, i}\right) \mathcal{C}^{-1} \widetilde{H}(t) d t\right| \\
& \leq c \lambda_{X}^{d-2 Q-2} \sum_{r=1}^{R} \sum_{j=1}^{K_{r}} \sum_{s=1}^{R} \sum_{i=1}^{K_{s}} a_{r, j} a_{s, i} \\
& \leq c X^{1-(2 Q+2) / d} \sum_{r=1}^{R} \sum_{s=1}^{R} \sum_{j=1}^{K_{r}} a_{r, j} \sum_{i=1}^{K_{s}} a_{s, i} \\
& \leq c X^{1-(2 Q+2) / d} \sum_{r=1}^{R} \sum_{s=r}^{R}\left(\sum_{j=1}^{K_{r}} a_{r, j}\right)^{2} \\
& \left.\leq c X^{1-(2 Q+2) / d} \sum_{r=1}^{R}(R-r+1) \sum_{j=1}^{K_{r}} a_{r, j}\right)^{2} \\
& \leq c \kappa X^{2-(2 Q+2) / d} \sum_{r=1}^{R}\left(\sum_{j=1}^{K_{r}} a_{r, j}\right)^{2}
\end{aligned}
$$

and since $Q>d+3$, the exponent $2-(2 Q+2) / d$ is negative and the result follows. 
Lemma 12 There exist $C>0$ and $X_{0}>0$ such that for every $X>X_{0}$

$$
\left|\sum_{r=1}^{R} \sum_{j=1}^{K_{r}} \sum_{s=1}^{R} \sum_{i=1}^{K_{s}} a_{r, j} a_{s, i} F_{5}\left(x_{r, j}, x_{s, i}\right)\right| \leq C \sum_{r=1}^{R}\left(\sum_{j=1}^{K_{r}} a_{r, j}\right)^{2} .
$$

Proof We need to estimate

$$
\begin{aligned}
& \left|\sum_{r=1}^{R} \sum_{j=1}^{K_{r}} \sum_{s=1}^{R} \sum_{i=1}^{K_{s}} a_{r, j} a_{s, i} \sum_{m=0}^{+\infty}\left(H\left(\frac{\lambda_{m}}{\lambda_{X}}\right)-\widetilde{H}\left(\lambda_{m}\right)\right) \varphi_{m}\left(x_{r, j}\right) \overline{\varphi_{m}\left(x_{s, i}\right)}\right| \\
& \quad \leq 2 \sum_{r=1}^{R} \sum_{j=1}^{K_{r}} \sum_{s=r}^{R} \sum_{i=1}^{K_{s}} \sum_{m=0}^{+\infty} a_{r, j} a_{s, i}\left|H\left(\frac{\lambda_{m}}{\lambda_{X}}\right)-\widetilde{H}\left(\lambda_{m}\right)\right|\left|\varphi_{m}\left(x_{r, j}\right)\right|\left|\varphi_{m}\left(x_{s, i}\right)\right| .
\end{aligned}
$$

Let us first study the term

$$
I(x)=H\left(\frac{x}{\lambda_{X}}\right)-\widetilde{H}(x)=\int_{\mathbb{R}^{d}}\left[H\left(\frac{x}{\lambda_{X}}\right)-H\left(\frac{x-y}{\lambda_{X}}\right)\right] \eta(y) d y .
$$

Since $\eta(y)$ has rapid decay at infinity and $H(x)$ is supported in $B(0,1)$, if $|x| \geq 2 \lambda_{X}$ we have

$$
\begin{aligned}
|I(x)| & \leq \int_{\mathbb{R}^{d}}\left|H\left(\frac{x-y}{\lambda_{X}}\right) \eta(y)\right| d y \leq \int_{\left\{|x-y| \leq \lambda_{X}\right\}}\left|H\left(\frac{x-y}{\lambda_{X}}\right) \eta(y)\right| d y \\
& \leq c \int_{\left\{|y| \geq|x|-\lambda_{X}\right\}}|\eta(y)| d y \leq C\left(1+|x|-\lambda_{X}\right)^{-M} .
\end{aligned}
$$

Assume $|x|<2 \lambda_{X}$. By Taylor's theorem with integral reminder we can write

$$
\begin{aligned}
& H\left(\frac{x}{\lambda_{X}}-\frac{y}{\lambda_{X}}\right) \\
& =H\left(\frac{x}{\lambda_{X}}\right)+\sum_{1 \leq|\alpha| \leq M-1} \frac{1}{\alpha !} \frac{\partial^{|\alpha|} H}{\partial x^{\alpha}}\left(\frac{x}{\lambda_{X}}\right)\left(-\frac{y}{\lambda_{X}}\right)^{\alpha} \\
& \quad+\sum_{|\alpha|=M} \frac{M}{\alpha !}\left(-\frac{y}{\lambda_{X}}\right)^{\alpha} \int_{0}^{1}(1-t)^{M-1} \frac{\partial^{|\alpha|} H}{\partial x^{\alpha}}\left(\frac{x}{\lambda_{X}}-t \frac{y}{\lambda_{X}}\right) d t
\end{aligned}
$$

so that

$$
\begin{aligned}
H\left(\frac{x}{\lambda_{X}}-\frac{y}{\lambda_{X}}\right)= & H\left(\frac{x}{\lambda_{X}}\right)+\sum_{1 \leq|\alpha| \leq M-1} \frac{1}{\alpha !} \frac{\partial^{|\alpha|} H}{\partial x^{\alpha}}\left(\frac{x}{\lambda_{X}}\right)\left(-\frac{y}{\lambda_{X}}\right)^{\alpha} \\
& +O\left(\left|-\frac{y}{\lambda_{X}}\right|^{M}\right) .
\end{aligned}
$$


It follows that

$$
\begin{aligned}
I(x)= & \int_{\mathbb{R}^{d}}\left[H\left(\frac{x}{\lambda_{X}}\right)-H\left(\frac{x-y}{\lambda_{X}}\right)\right] \eta(y) d y \\
= & -\sum_{1 \leq|\alpha| \leq M-1} \frac{1}{\alpha !} \frac{\partial^{|\alpha|} H}{\partial x^{\alpha}}\left(\frac{x}{\lambda_{X}}\right) \int_{\mathbb{R}^{d}}\left(-\frac{y}{\lambda_{X}}\right)^{\alpha} \eta(y) d y \\
& +\int_{\mathbb{R}^{d}} O\left(\frac{|y|^{M}}{\lambda_{X}^{M}}\right) \eta(y) d y
\end{aligned}
$$

and since

$$
\int_{\mathbb{R}^{d}} y^{\alpha} \eta(y) d y=\mathcal{F}_{d}\left(y^{\alpha} \eta(y)\right)(0)=(-2 \pi i)^{-|\alpha|} \frac{\partial^{|\alpha|} \mathcal{F}_{d} \eta}{\partial \xi^{\alpha}}(0)=0
$$

we obtain

$$
|I(x)| \leq c \lambda_{X}^{-M}
$$

Using Hormander's estimates on the $L^{\infty}$ norm of the eigenfunctions (see [16, (3.2.2), page 48])

$$
\left\|\varphi_{m}\right\|_{\infty} \leq C\left(1+\lambda_{m}\right)^{\frac{d-1}{2}}
$$

we obtain

$$
\begin{aligned}
& \sum_{r=1}^{R} \sum_{j=1}^{K_{r}} \sum_{s=r}^{R} \sum_{i=1}^{K_{s}} \sum_{m=0}^{+\infty} a_{r, j} a_{s, i}\left|H\left(\frac{\lambda_{m}}{\lambda_{X}}\right)-\widetilde{H}\left(\lambda_{m}\right)\right|\left|\varphi_{m}\left(x_{r, j}\right)\right|\left|\varphi_{m}\left(x_{s, i}\right)\right| \\
& \leq c \lambda_{X}^{-M} \sum_{\lambda_{m} \leq 2 \lambda_{X}}\left(1+\lambda_{m}\right)^{d-1} \sum_{r=1}^{R} \sum_{s=r}^{R} \sum_{j=1}^{K_{r}} a_{r, j} \sum_{i=1}^{K_{s}} a_{s, i} \\
& \quad+c \sum_{\lambda_{m} \geq 2 \lambda_{X}}\left(1+\lambda_{m}-\lambda_{X}\right)^{-M} \lambda_{m}^{d-1} \sum_{r=1}^{R} \sum_{s=r}^{R} \sum_{j=1}^{K_{r}} a_{r, j} \sum_{i=1}^{K_{s}} a_{s, i} \\
& \left.\quad \leq c\left(\lambda_{X}^{-M} \sum_{\lambda_{m} \leq 2 \lambda_{X}}\left(1+\lambda_{m}\right)^{d-1}+\sum_{\lambda_{m} \geq 2 \lambda_{X}} \lambda_{m}^{-M+d-1}\right) \sum_{r=1}^{R}(R-r+1) \sum_{j=1}^{K_{r}} a_{r, j}\right)^{2} \\
& \quad \leq c \kappa X\left(\lambda_{X}^{-M} \sum_{\lambda_{m} \leq 2 \lambda_{X}}\left(1+\lambda_{m}\right)^{d-1}+\sum_{\lambda_{m} \geq 2 \lambda_{X}} \lambda_{m}^{-M+d-1}\right) \sum_{r=1}^{R}\left(\sum_{j=1}^{K_{r}} a_{r, j}\right)^{2} .
\end{aligned}
$$


By Weyl's estimates, which say that the number of eigenvalues $\lambda_{m}^{2} \leq T^{2}$ is asymptotic to $c T^{d}$,

$$
\begin{aligned}
& \lambda_{X}^{-M} \sum_{\lambda_{m} \leq 2 \lambda_{X}}\left(1+\lambda_{m}\right)^{d-1}+\sum_{\lambda_{m} \geq 2 \lambda_{X}} \lambda_{m}^{-M+d-1} \\
& \leq c \lambda_{X}^{-M} \lambda_{X}^{d} \lambda_{X}^{d-1}+\sum_{k=1}^{+\infty} \sum_{2^{k} \lambda_{X} \leq \lambda_{m} \leq 2^{k+1} \lambda_{X}} \lambda_{m}^{-M+d-1} \\
& \leq c \lambda_{X}^{-M+2 d-1}+c \sum_{k=1}^{+\infty} 2^{d(k+1)} \lambda_{X}^{d}\left(2^{k} \lambda_{X}\right)^{-M+d-1} \\
& \leq c \lambda_{X}^{-M+2 d-1}+c \lambda_{X}^{-M+2 d-1} \sum_{k=1}^{+\infty} 2^{(2 d-M-1) k}
\end{aligned}
$$

and taking $M$ such that $-M+2 d-1<-d$ gives the result.

Funding Open access funding provided by Università degli Studi di Bergamo within the CRUI-CARE Agreement

Open Access This article is licensed under a Creative Commons Attribution 4.0 International License, which permits use, sharing, adaptation, distribution and reproduction in any medium or format, as long as you give appropriate credit to the original author(s) and the source, provide a link to the Creative Commons licence, and indicate if changes were made. The images or other third party material in this article are included in the article's Creative Commons licence, unless indicated otherwise in a credit line to the material. If material is not included in the article's Creative Commons licence and your intended use is not permitted by statutory regulation or exceeds the permitted use, you will need to obtain permission directly from the copyright holder. To view a copy of this licence, visit http://creativecommons.org/licenses/by/4.0/.

\section{References}

1. Berger, M., Gauduchon, P., Mazet, E.: Le spectre d'une variété riemannienne. Lecture Notes in Mathematics, vol. 194. Springer, Berlin (1971)

2. Bilyk, D., Dai, F.: Geodesic distance Riesz energy on the sphere. Trans. Am. Math. Soc. 372(5), 3141-3166 (2019)

3. Bilyk, D., Dai, F., Steinerberger, S.: General and refined Montgomery lemmata. Math. Ann. 373, 1283-1297 (2019)

4. Bondarenko, A., Radchenko, D., Viazovska, M.: Optimal asymptotic bounds for spherical designs. Ann. Math. 178, 443-452 (2013)

5. Cassels, J.W.S.: On the sums of powers of complex numbers. Acta Math. Acad. Sci. Hungar. 7, 283-289 (1956)

6. Ehler, M., Etayo, U., Gariboldi, B., Gigante, G., Peter, T.: Asymptotically optimal cubature formulas on manifolds for prefixed weights (2020). arXiv:1810.01106v3

7. Erdélyi, A., Magnus, W., Oberhettinger, F., Tricomi, F. G.: Tables of integral transforms, vol. I. Based, in part, on notes left by Harry Bateman. McGraw-Hill Book Company, Inc., New York (1954)

8. Gariboldi, B., Gigante, G.: Optimal asymptotic bounds for designs on manifolds. arXiv:1811.12676

9. Gel'fand, I.M., Shilov, G.E.: Generalized Functions, vol. 1. Academic Press, London (1964)

10. Gigante, G., Leopardi, P.: Diameter bounded equal measure partitions of Ahlfors regular metric measure spaces. Discrete Comput. Geom. 57, 419-430 (2017)

11. Hormander, L.: The Analysis of Linear Partial Differential Operators, I, II, III, IV. Springer (19831985) 
12. Korevaar, J., Meyers, J.L.H.: Spherical Faraday cage for the case of equal point charges and Chebyshevtype quadrature on the sphere. Integr. Transform. Spec. Funct. 1, 105-117 (1993)

13. Lu, J., Steinerberger, S.: Riesz Energy on the Torus: Regularity of Minimizers (2017). arXiv: 1710.08010

14. Montgomery, H.: Ten Lectures at the Interface of Harmonic Analysis and Number Theory. American Mathematical Society, Providence (1994)

15. Siegel, C.L.: Über Gitterpunkte in Convexen Körpern und ein Damit Zusammenhängendes Extremalproblem. Acta Math. 65(1), 307-323 (1935)

16. Sogge, C.D.: Hangzhou Lectures on Eigenfunctions of the Laplacian, Annals of Mathematics Studies, 188. Princeton University Press, Princeton (2014)

17. Stein, E.M., Weiss, G.: Introduction to Fourier analysis on Euclidean spaces. Princeton University Press, Princeton (1971)

18. Steinerberger, S.: Spectral limitations of quadrature rules and generalized spherical designs. Int. Math. Res. Notices. https://doi.org/10.1093/imrn/rnz176

19. Stempak, K., Trebels, W.: Hankel multipliers and transplantation operators. Stud. Math. 126, 51-66 (1997)

20. Travaglini, G.: Number Theory, Fourier Analysis and Geometric Discrepancy, London Mathematical Society Student Texts, vol. 81. Cambridge University Press, Cambridge (2014)

Publisher's Note Springer Nature remains neutral with regard to jurisdictional claims in published maps and institutional affiliations. 